\title{
Re: Validation of cross-sectional studies with longitudinal meta-analysis
}

\author{
J.-a. Liu $^{1} \cdot$ Q. Wang ${ }^{2}$
}

Received: 29 February 2016/Accepted: 30 March 2016/Published online: 25 April 2016

(C) International Osteoporosis Foundation and National Osteoporosis Foundation 2016

\section{Dear Editor,}

We thank Drs. Cure-Cure and Cure [1] for their interest in our recently published article [2]. We agree that well-designed cross-sectional studies are early, reliable, and cost-effective sources of information. Meta-analysis of high-quality crosssectional studies should be encouraged. This analysis will facilitate the presentation of valuable and reliable evidence combined with findings of prospective studies.

In our previous meta-analyses, we searched for prospective and non-prospective (e.g., cross-sectional and case control) studies reporting the parity-related risk of osteoporotic fracture (OF) at any skeletal location, such as spine, wrist, and hip. Subgroup analysis based on non-prospective reports indicated that parous women exhibited a lower OF risk than nulliparous women, with the corresponding pooled OR of 0.725 (95\% confidence interval $\left.(\mathrm{CI})=0.614-0.836, I^{2}=57.3 \%, n=19\right)$, $0.803\left(95 \% \mathrm{CI}=0.730-0.876, I^{2}=0.0 \%, n=15\right)$, and 0.612 $\left(95 \% \mathrm{CI}=0.490-0.733, I^{2}=47.1 \%, n=14\right)$ for at least $1,1-$ 2 , and 3 or more parities, respectively. When the hip fracture risk was exclusively considered, the respective counterpart values were $0.797\left(95 \% \mathrm{CI}=0.717-0.877, I^{2}=0.0 \%\right.$, $n=12), 0.835\left(95 \% \mathrm{CI}=0.725-0.944, I^{2}=0.0 \%, n=10\right)$, and $0.726\left(95 \% \mathrm{CI}=0.610-0.843, I^{2}=0.0 \%, n=10\right)$.

J.-a. Liu

lja10_11@163.com

1 Department of Social Medicine and Health Management, School of Public Health, Tongji Medical College, Huazhong University of Science and Technology, Wuhan, Hubei Province, China

2 Department of Epidemiology and Biostatistics, School of Public Health, Tongji Medical College, Huazhong University of Science and Technology, Wuhan, Hubei Province, China
We hypothesize that the specific explanatory variable of interest (i.e., parity) contributes to the concurrent findings from prospective and non-prospective studies. Based on Hill's criteria for epidemiologic causal inference, time sequence of cause and effect is legitimate. That's the major limitation of cross-sectional study. Generally, deliveries occur before menopause, and women often suffer OF after menopause. Therefore, it's reasonable to assume that most OFs among women occur after delivery. That's why crosssectional studies may also present reliable information on parity-related OF risk.

\section{Compliance with ethical standards}

Conflict of interest None.

\section{References}

1. Cure-Cure C, Cure-Ramirez P (2001) Hormone replacement therapy for bone protection in multiparous women: when to initiate it. Am J Obstet Gynecol 184(4):580-583

2. Wang Q, Huang Q, Zeng Y, Liang J, Liu S, Gu X, Liu J (2016) Parity and osteoporotic fracture risk in postmenopausal women: a doseresponse meta-analysis of prospective studies. Osteoporosis Int 27: 319-330. doi:10.1007/s00198-015-3351-3 